\title{
ADMS4LV - advanced distribution management system for active management of LV grids
}

\author{
Filipe Campos ${ }^{1} \bowtie$, Luís Marques ${ }^{1}$, Nuno Silva ${ }^{1}$, Francisco Melo ${ }^{2}$, \\ Luís Seca ${ }^{3}$, Clara Gouveia ${ }^{3}$, André Madureira ${ }^{3}$, Jorge Pereira ${ }^{4}$ \\ ${ }^{1}$ SmartGrids R\&D, Efacec, Maia, Portugal \\ ${ }^{2} D P D R$, EDP Distribuição, Lisboa, Portugal \\ ${ }^{3}$ Centre for Power and Energy Systems, INESC TEC, Porto, Portugal \\ ${ }^{4}$ Power Systems Unit, INESC TEC, Porto, Portugal \\ $凶$ E-mail: fcampos@efacec.com
}

\begin{abstract}
This study describes the main developments of the advanced distribution management system for active management of low-voltage (ADMS4LV) Grids project -. ADMS4LV is a R\&D project that targets the development and demonstration of a system with adequate tools to monitor, control and manage the LV networks with the objective of an effective implementation of smart grids (SG). The main architecture of ADMS4LV is detailed, and the main functionalities of its modules are described. The adequacy of ADMS4LV is validated using a large-scale demonstrator that is based on a typical Portuguese LV network, where there are already present some of the features of an SG, namely an advanced metering infrastructure and distributed energy resources. This project gathers partners with different profiles such as the Portuguese DSO (EDP-D) and one of the major R\&D institutes in the area of energy (INESC-TEC).
\end{abstract}

\section{Introduction}

The role of distribution networks (DNs) in the overall system's operation is changing. The deployment of smart grid (SG) concepts and the large-scale integration of distributed energy resources (DER) is changing the philosophy of DN's planning and operation.

The majority of the changes are occurring at the LV networks with the integration of micro-generation, grid storage systems, electric vehicles $(\mathrm{EV})$ and the deployment of demand side management strategies as well as self-consumption concepts. In this sense, new solutions are required in order to ensure a flexible, reliable and efficient operation of these networks.

However, the management and operation of LV networks can be quite challenging, considering their extension and the number of assets to control, when compared to higher voltage levels. In addition, some of these networks may have several decades of lifetime, and therefore, their characterisation (e.g. branches' physical characteristics) might be insufficient or affected by gross errors. Furthermore, the monitoring capabilities at the LV network are typically reduced.

At the same time, the current introduction of advanced metering infrastructures (AMIs) in LV networks provide relevant data for improving the awareness and efficiency of the system, namely, a set of electrical variables as voltages at consumer's premises and consumers' load profiles at regular time periods (e.g. $15 \mathrm{~min}$ periods). This information together with new monitoring and control equipment designed specifically for LV network increases the system observability and consequently the DSO's capability to monitor and control LV networks. Nevertheless, due to the absence of effective solutions to manage AMI data in an efficient and smart manner, they have been mainly used for billing and planning purposes.

Today the most relevant distribution management system (DMS) suppliers have recognise the need of providing new solutions for distribution networks management and operation, such as the ones proposed in $[1,2]$, presenting new systems and network applications capable of dealing with DER and managing smart metering data for operation and outage detection purposes, considering their interaction with outage management systems. However, the majority of these systems are based on the extension of the current and traditional tools used in the voltages levels above, being mainly focused in the demand side management. In fact, the solutions found in the market are not suitable for the specificities of LV networks since are required approaches exclusively designed for them. For instance, in some of the systems proposed power flow and state estimation algorithms for LV networks are similar to those used for the high voltage (HV) and medium voltage (MV) networks.

This paper presents the ADMS4LV Grids, which aims at providing an innovative monitoring, management and control solution for the LV networks of the future. By handling the information in a smart and efficient manner and using several advanced algorithms, specifically designed for LV networks, its main objective is to enhance the operation of these networks, contributing therefore, to a more efficient, secure and reliable operation of the distribution network.

\section{System overview and description}

The ADMS4LV is a solution for the DSO command centre for the LV network. Therefore, it is designed to manage the data from AMI infrastructure for LV network monitoring and control purposes as well as work in coordination with higher hierarchical level systems (corporative systems - back office), namely those which have tools already developed specifically for MV networks.

The AMI architecture is based on InovGrid project [3], comprising the comprising smart meters (SMs) at the majority of consumers' locations, intelligent electronic device (IEDs), network smart sensors, whose information is collected and pre-processed by the smart controllers at the secondary substation (SS) - the distribution transformer controller (DTC).

Fig. 1 depicts an overview of the ADMS4LV architecture where are present its core functionalities that will give rise to several 


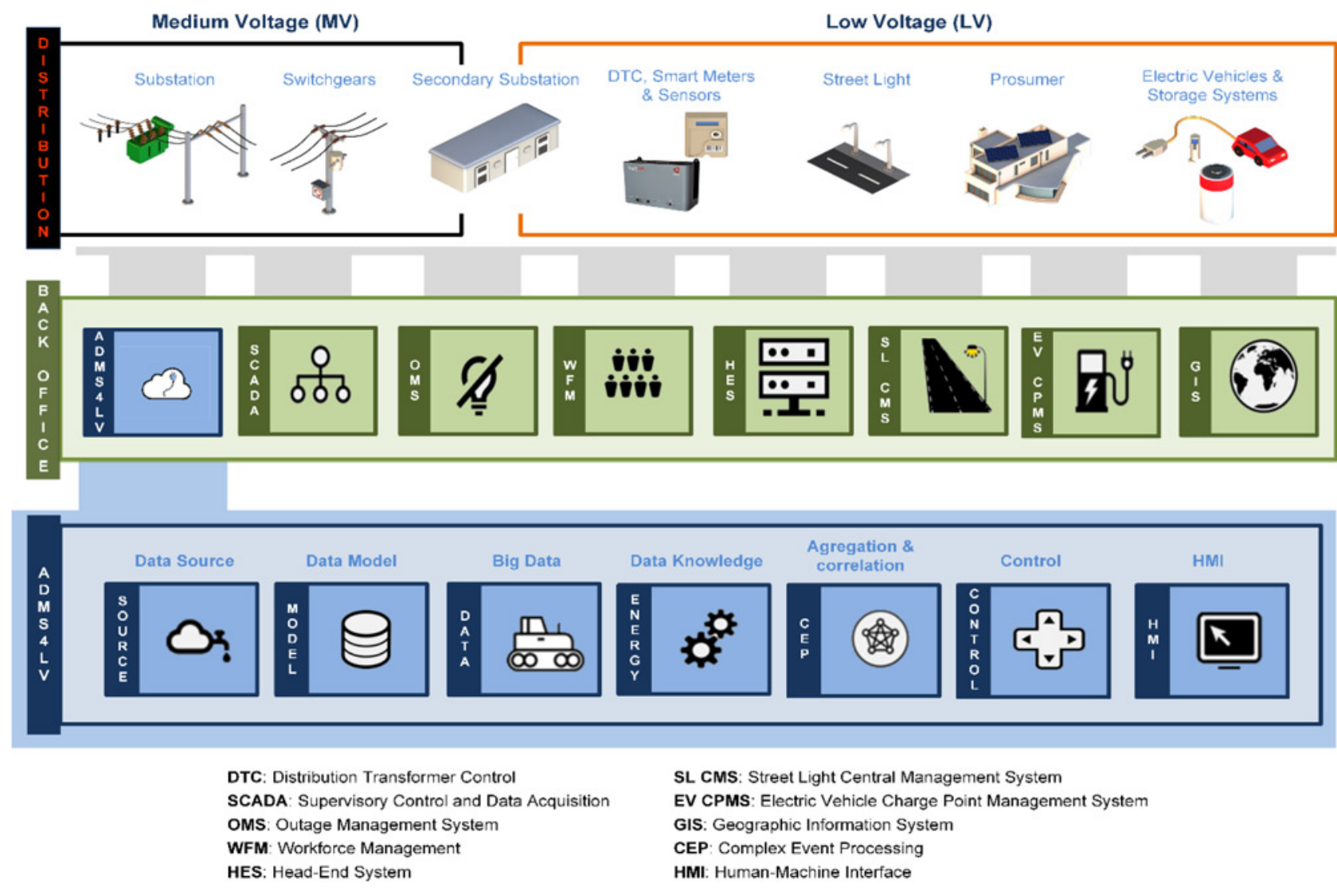

Fig. 1 ADMS4LV architecture

advanced modules for network's management. Through the manipulation of high quantities of data (Big Data) using processing techniques (aggregation and correlation) to extract relevant features that, combined with the known characteristics of the network (data model) and the relations between the electric variables (data knowledge), is possible to infer about the real operation conditions of the network and provide automatic controls to maintain its safety and efficient operation. The most relevant modules constituting ADMS4LV are described below.

\subsection{Module for data management and processing}

This module will be the backbone of the ADMS4LV as it will be responsible for all the tasks that involve the treatment of the data received from the field for operation purposes, namely from network monitoring equipment and the smart metering infrastructure. The module will be responsible for:

- Communications' state monitoring - responsible for assessing the connectivity between the SMs and the DTC and the signal strength in order to prevent failures and solve communication problems.

- Customers phase identification and topology processor - as previously mentioned, often the DSO does not have all the data available about the network and one of the most important concerns is regarding the identification of the phase where each customer is connected. This situation hinders the application of other important tools such as power flow algorithms. Thus, this module will use Big Data tools for applying regression and correlation techniques to infer about the phase connection of single-phase consumers, using time series measurements and geographical information.

- State estimation - since one of the goals of ADMS4LV is to increase the DSO's awareness about the network operation conditions, this tool will be responsible for providing a reliable state operation of the network. Based on artificial intelligence techniques [4], this algorithm is able to estimate the network state with limited knowledge of the network topology and characteristics and a reduced number of real-time measurements. The algorithm is based on the work developed under the scope of the supervisory control and data acquisition for low voltage (SCADABT) project [5]. The algorithm is able to estimate the state of the network, based on the measurements collected in real-time from a reduced number of IEDs or SMs. The group of IEDs and SMs communicating in real-time will dynamically change according to their location, the network topology and location of flexible DER. This will allow to maximise the network's observability, while minimising the number of measurements required;

- Checking data quality and consistency. One of the main challenges of using AMI data for operation purposes is the need of adequate mechanisms to process the data collected and build a consistent historical database. This module will include algorithms responsible for verifying the data consistency by identifying outliers (erroneous measurements) and to perform imputation (replacing missing values).

Finally, taking into account the computational effort that may be required by this module, some of these functionalities will be implemented using compute unified device architecture. Algorithms that are implemented in graphical processing units, instead of central processing units, allows a significantly increase of computing performance.

\subsection{Module for event correlation and aggregation}

One of the main challenges of a system dedicated to the LV network, is the need to deal efficiently with high amounts of data, such as events and alarms generated by the different IEDs and SMs connected at the LV level. One example of this situation is the occurrence of a blackout in a certain zone of the network: all the devices located downstream will become offline, generating therefore a considerable amount of events indicating communication failures. Thus, this module will be responsible for 
receiving information from different systems (both at field and back office levels), and network applications and infer about the origin of the events and proceed in conformity, generating relevant information for the network operator. This is accomplished by using techniques of correlation and aggregation that will make possible, in a systematic way, achieve the following results:

- Process events and categorise them according to their origin (e.g. number of communication failures between the DTC and supervisory control and data acquisition; number and duration of over/under voltages situations).

- Infer about the probable cause and location of the main event that generated the cascade failure.

- Generate alarms and categorise it according to their severity and/ or relevance for the network's operation.

This module will be also responsible for calculating, in an automatic manner, reliability indices, according to the nature of events.

This module is a key element of the ADMS4LV, since it is responsible for increasing the monitoring capability of the system. Based on the information provided, the operator will be able to define adequate operation and planning procedures to improve the system efficiency, power quality and continuity of service, supported by innovative network applications.

\subsection{Module for preventive management of $L V$ networks considering the integration of DER}

AMI provides relevant information for LV operation purposes, namely node voltages and load diagrams. Such information, allows to build relevant historical databases which can be used to characterise load behaviour either through forecasts or innovative load allocation techniques.

Based on this information, this module will implement a preventive management of LV networks, studying the expected network state a priori in order to identify potential technical problems in the network, namely those resulting of the large-scale deployment of DER. In fact, the large scale deployment of DER, such as EV and micro generation units can pose several challenges for the technical operation of the LV network, namely in what concerns voltage and branch loading [6]. However, the deployment of home energy management systems together with self-consumption schemes, changes the expected behaviour of loads and micro-generation, increasing the flexibility of the consumers' load.

The module will evaluate the network state for the next hours or day and define the most adequate control actions, which ensure the secure operation of the system, taking advantage of both LV network controllable assets and the flexibility of consumers. The following resources will be considered:

- on load tap changing at the SS,

- LV switching equipment, enabling network reconfiguration,

- energy storage systems,

- public charging infrastructures,

- aggregated flexibility of LV consumers.

The network state namely voltages and branch loadings will be determined through a smart power flow algorithm, designed for unbalanced three-phase four wire unbalanced networks. The control actions will then be determined the impact of the different DER units in the location where the violation is observed, using voltage sensitivity factors.

Considering the uncertainty associated to the customers' behaviour, the control actions will be updated considering the real-time network operation state determined by the state estimation algorithm running online. This will ensure that the plan define will actually optimise the network operation and not compromise its security.

\subsection{Module of loss characterisation and non-technical loss (NTL) detection}

One of the greatest impacts on the network's efficiency regards to the total energy of losses, including the technical and NTLs. NTLs represent all the energy that is supplied, without being paid either dissipated in the systems' infrastructures (technical losses). NTLs are most of all caused by frauds and non-detected high impedance faults [7]. For this purpose has been developed a hybrid approach by incorporating simultaneously two different but complementary fields: artificial intelligence and the electrical energy theory. The major advantages of this tool regards to its high scalability and adaptability: it adapts the calculations and the data used according to the particularities of each LV network minimising the computational effort and the amount of SMs measurements required.

2.4.1 Read data: The data used as input encompasses: Energy Records - historic records of consumers' SMs and DTCs, namely active and reactive measurements and voltages; network data technical characteristics about the network. According to the network, data is possible to define two different scenarios that will lead to two different analysis:

- scenario 1: full knowledge of the grid topology,

- scenario 2: partial or total absence of knowledge about the network.

2.4.2 Network loss characterisation: In scenario 1 it is possible to carry out power flow studies giving as output statistics about the per phase losses per network section (e.g. for each line/ branch) as well as the technical losses of the overall network (TLP). It is also possible to identify busbars with high load imbalances that may lead to high neutral currents. In case the scenario 2 is observed, it is only possible to produce statistics about the real total losses (RTL), i.e. difference between the total energy supplied by the SS and the aggregated consumption.

2.4.3 NTLs detection: This step is used to infer about the presence of NTLs in the network and their localisation is performed only in case their presence is considered suspicious. For scenario 1 this is performed by comparing the RTL with the TLP for each phase. For scenario 2 the detection of NTLs is performed by comparing the RTL with a typical value for the network losses extract, transform and load (ETLs) based on the features of the network under consideration (e.g. number of consumers, type of network) [8]

2.4.4 NTLs localisation: The fraudulent consumers are identified by using a machine-learning algorithm that classifies consumers according to the patterns of their load profiles. Notwithstanding in scenario 1 this is only performed for the consumers connected to the phases in which the presence of NTLs have been considered suspicious.

2.4.5 Report to DSO: In the end of the process are reported to the DSO all the statistics about the losses behaviour and the locations where the presence of NTLs are suspicious. In that way is possible to send crews for the terrain in order to proceed to onsite inspections.

\subsection{Module for EV charging stations management}

The implementation of smart charging strategies may avoid the need of network reinforcement investments. The EV may be regarded as a flexible load, limited to the owner charging schedule and preferences. Following the preventive LV network operation strategy, this module will schedule and control public and possibly private EV charging points, according to the network capacity to accommodate the load associated to EV charging.

Taking into account the expected charging profiles as well as the EV charging requirements, the module will indicate for the next hours/day the charging points which are available/unavailable for 


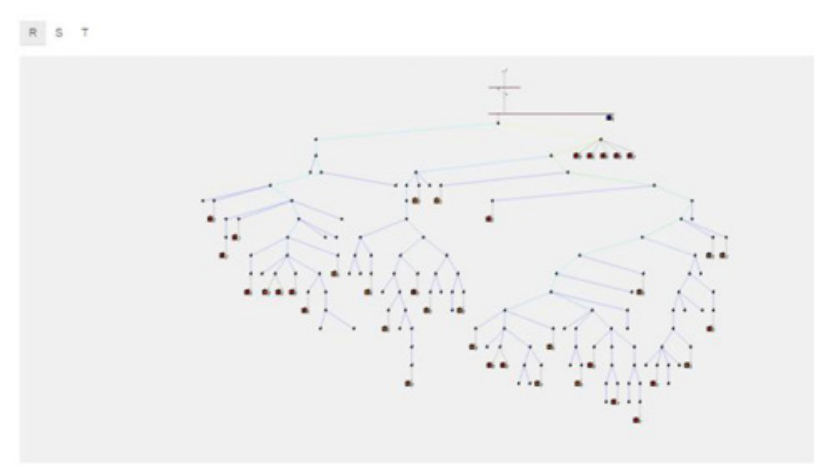

Fig. 2 Example of web HMI for power flow

charging or with limited charging power, and manage it in real time according to the defined information.

This module will be a module that can be installed at a DSO, whew national charge point management infrastructures can request a charging availability.

\subsection{Module: enhanced user interface}

One of the concerns of ADMS4LV is to facilitate network operation, by supporting the DSO in the decision-making. Thus, there will be available a graphical user interface where will be displayed all the relevant information about the network and its state operation in a user-friendly manner. This module provides, therefore, two ways of support for the DSO: on one hand, the information will be displayed via human machine interface (HMI) web, comprising the networks synoptic with the geolocation of the system's infrastructures. It will allow a reliable and simplified vision of the network, comprising several information to aid the operators controlling the network. On the other hand, a mobile application will be developed incorporating augmented reality functionalities that will be used to support the crews on the terrain as exemplified in Fig. 2.

\section{Pilot demonstration site}

The functionalities described in this paper developed under the scope of the ADMS4LV will be target of a rigorous assessment and validation through tests in laboratory and in a real large-scale pilot demonstrator. The validation of each specific module is performed using a set of use cases, which have been defined by the Portuguese DSO EDPD. The target pilot targets a minimum of 3000 SMs with power line carrier (PLC) communication, on rural and urban LV networks, with DER and EV integration.

\section{Conclusions}

In this paper, an overview of the ADMS4LV, consisting of a monitoring, management and control system fully designed for the operation of LV networks considering its specific characteristics and the gradual integration of DER and new demand side management services is presented. More than a system to manage the heterogeneous assets present in LV networks, ADMS4LV provides effective solutions to operate these networks in a preventive manner, anticipating future problems, notifying the operator in an effective manner and providing the adequate control actions for operating the system.

Due to some possible lack of characterisation of LV network, the manner of managing and selecting the data according to the different needs is one of the cornerstones of ADMS4LV - using the most relevant measurements, it guarantees the observability of the network while reducing the amount of data required, and therefore, the computational effort. On the other hand, ADMS4LV uses communication standards and protocols, guaranteeing interoperability, which is of the utmost importance in order to achieve an effective implementation of the smart grid concept. In addition, there is also interoperability between the new proposed tools, under the scope of this project, and corporative systems (back office) - making this system adequate for large-scale deployment.

Finally, the ongoing pilot project validates the adequacy of ADMS4LV, making it as a turnkey solution for the management of DNs. In fact, the ADMS4LV brings new tools and functionalities that go beyond the state of the art, creating a benefit solution.

\section{References}

1 Schneider: 'Advanced distribution management system (ADMS)', Available: http://www.schneider-electric.com/products/ww/en/1900-feederautomation/1995advanced-distribution-management-system/61751-adms/, accessed: August 2016

2 ABB: MicroSCADA Pro. Available: http://www.abb.com/product/db0003db004281/ c12573990068e 57cc1256ebc005454be.aspxproductLanguage $=$ us \& country $=$ PT, accessed August 2016

3 Cunha, L.: 'InovGrid project - distribution network evolution as a decisive answer to new electrical sector challenges', 2008

4 Barbeiro, P. N. P., Krstulovic, J., Teixeira, H., et al.: 'State estimation in distribution smart grids using autoencoders'. IEEE 8th Int. Power Engineering and Optimization Conf. (PEOCO), Langkawi, 2014

5 Silva, N., Silva, P. M., Seca, L., et al.: 'LV SCADA - how to effectively manage LV networks with limited topology and electrical characteristics data'. 23rd Int. Conf. on Electricity Distribution, Cired, 2015

6 Madureira, A., Seca, L., Lopes, J.P.: 'Coordinated voltage control in distribution systems under the smart grid concept'. Proc. CIRED 2012 Workshop, Integration of Renewables into the Distribution Grid, 2012, vols. 1-4

7 Marques, L.: 'Detection and location of non-technical losses in low voltage distribution networks'. Master dissertation, FEUP, Porto, 2016

8 Silva, N.: 'Alternative design strategies of distribution systems'. PhD thesis, Imperial College of London, University of Manchester, 2009 